\title{
Interventions for postnatal depression assessing the mother-infant relationship and child developmental outcomes: a systematic review
}

This article was published in the following Dove Press journal:

International Journal of Women's Health

23 April 2015

Number of times this article has been viewed

\author{
Zoe-Lydia Tsivos' \\ Rachel Calam' \\ Matthew R Sanders ${ }^{1,2}$ \\ Anja Wittkowski' \\ 'School of Psychological Sciences, \\ University of Manchester, Manchester, \\ UK; ${ }^{2}$ Parenting and Family Support \\ Center, University of Queensland, \\ Brisbane, Australia
}

\begin{abstract}
Postnatal depression (PND) has negative effects on maternal well-being as well as implications for the mother-infant relationship, subsequent infant development, and family functioning. There is growing evidence demonstrating that PND impacts on a mother's ability to interact with sensitivity and responsiveness as a caregiver, which may have implications for the infant's development of self-regulatory skills, making the infant more vulnerable to later psychopathology. Given the possible intergenerational transmission of risk to the infant, the mother-infant relationship is a focus for treatment and research. However, few studies have assessed the effect of treatment on the mother-infant relationship and child developmental outcomes. The main aim of this paper was to conduct a systematic review and investigate effect sizes of interventions for PND, which assess the quality of the mother-infant dyad relationship and/or child outcomes in addition to maternal mood. Nineteen studies were selected for review, and their methodological quality was evaluated, where possible, effect sizes across maternal mood, quality of dyadic relationship, and child developmental outcomes were calculated. Finally, clinical implications in the treatment of PND are highlighted and recommendations made for further research.
\end{abstract}

Keywords: postnatal depression, infant development, intervention, dyad, mother-infant relationship, systematic review

\section{Introduction}

Approximately one in ten women suffers from postnatal depression (PND). ${ }^{1-3} \mathrm{Beck}^{4}$ reported that the best predictor of PND was depression in the antenatal period. A recent review identified a number of postnatal factors placing women at increased risk to continued depressive symptoms, including younger maternal age, poor education attainment, historical episodes of depression, antidepressant use during pregnancy, child developmental problems, low parental self-efficacy, poor relationship, and the occurrence of stressful life events. ${ }^{5}$ Psychosocial factors (ie, poverty, marital discord, life stressors) are thought to be more predictive of vulnerability to PND than biological or hormonal causes. ${ }^{1}$

PND has varied onset, chronicity, clinical presentation, and course relative to major depression and other mood disorders in the postpartum period, including postnatal blues and puerperal psychosis. ${ }^{6}$ Biopsychosocial models highlight the complexity and interaction between multiple systems implicated in PND. The model by Milgrom et $\mathrm{al}^{1}$ details vulnerability factors, precipitating factors (including those factors which may trigger PND onset: stress levels, stress-moderating variables of social support, and coping skills), and biological factors. The model also explains that sociocultural
Correspondence: Anja Wittkowski School of Psychological Sciences, 2nd Floor Zochonis Building, University of Manchester, Brunswick Street, Manchester, MI3 9PL, UK

Tel +44 I6I 3060400

Email anja.wittkowski@manchester.ac.uk 
factors may play a role in exacerbating and maintaining PND and they account for heterogeneity in vulnerability to experiencing PND across women.

An episode of PND generally lasts from 2 months to 6 months in duration and as long as 1 year in some cases. ${ }^{2,6,7}$ According to the Diagnostic and Statistical Manual of Mental Disorders (DSM), fourth edition, women meet diagnostic criteria for PND if the onset is within the first 4 weeks postpartum, although this onset period has been extended in clinical practice with reports that $50 \%$ of cases start within 3 months and $75 \%$ of cases within 7 months. ${ }^{8}$ The revised criteria in the DSM-V included a new specifier of mood episodes beginning in pregnancy. ${ }^{9}$ These criteria make limited reference to the infant, although the need for recognition of symptoms relating specifically to birth, labor, and other aspects of being a new parent has been identified elsewhere. ${ }^{10}$

The estimated cost of care alone between women with PND and those without PND in a British community sample is significant, reaching a mean cost of $£ 505.70$ for mothers without PND and $£ 786.20$ for mothers with PND a significant $(P=0.01)$ mean cost difference of $£ 280.50 .{ }^{11}$ Petrou et $\mathrm{al}^{11}$ have recommended that these are conservative estimates and that the excess cost is substantially more for women experiencing extended episodes of PND. Notwithstanding, there are further cost implications in terms of child and adolescent services accessed due to the increased risk associated with having a parent with PND. Treatment is, therefore, a major public health concern and the one which spans both maternal and infant mental health. ${ }^{12}$

Considerable evidence suggests that PND has profound and widespread effects on the mother, ${ }^{13}$ the mother-infant relationship, ${ }^{14,15}$ and serious implications for subsequent infant development ${ }^{16-18}$ and family well-being. ${ }^{19}$ Research has established that PND can affect the quality of parenting. ${ }^{20-22}$ In the context of PND, difficulties in practical parenting practices related to breastfeeding, ${ }^{23-25}$ sleep, ${ }^{26-28}$ infant health care, ${ }^{29}$ and safety practices ${ }^{24,25,30,31}$ have been reported. Following an episode of PND, women are predisposed to future risk of depressive episodes with subsequent children. Crucially, the first year is an important period for infants to develop self-regulatory skills. ${ }^{32,33}$ Adaptive development of self-regulatory skills in the infant is promoted by sensitive and responsive caregiving. PND directly impacts on a mother's ability to sensitively respond to her baby; thus, the quality of the dyadic relationship is also affected.

Interventions focusing exclusively on maternal depression may not be sufficient alone to buffer against the risks to infant development. ${ }^{34-36}$ In many instances, maternal mood may improve, but the intergenerational transmission of risk may continue to manifest. Conceptualizing the depressive episode within the context of the perinatal period may promote adaptive developmental pathways in the infant. It is therefore necessary to measure outcomes in order to understand if interventions for PND exert a protective effect on the mother-infant relationship and infant development in addition to maternal mood.

\section{Intergenerational transmission of risk to children of women with PND}

Goodman and Gotlib ${ }^{37}$ highlighted the need for a developmental model, which explains the transmission and manifestation of vulnerability in infants. The nature of the association between PND and infant development is especially complicated by limited understanding of the full impact and risk of maternal mood and cognitions on infant developmental pathways.

In their integrated model, Goodman and Gotlib ${ }^{37}$ detail how the effects of PND are implicated across the intergenerational gap. The model reflects the complex interplay between quality of parenting and several mechanisms (ie, heritability of depression, neuroregulatory mechanisms, exposure to negative maternal cognitions, behavior and affect, and sociodemographic conditions) and moderating risk factors (ie, paternal health/involvement, course and timing of depression, and individual child characteristics) which influence the developing infant (see Goodman and Gotlib $^{37}$ for a description of the model).

\section{Effect of PND on infant development}

Evidence suggests that PND in the parent may contribute to serious effects on infant cognitive and emotional development and is associated with later psychopathology and atypical development. ${ }^{38-40,44}$

Grace et $\mathrm{al}^{41}$ highlighted that the most significant effects of PND were on cognitive development including language development and intelligence. However, effects varied with characteristics of children involved, including sex and contextual factors as indicated by the aforementioned model. They also suggested that timing and course of PND were more pervasive in their effects on child development.

Research using the face-to-face video interaction paradigm has demonstrated that mothers with PND are more negative and their infants less positive than nondepressed mother-infant dyads. ${ }^{42}$

Longitudinal studies have also shown a predictive link between early PND and problems much later in 
development. ${ }^{17,43}$ Milgrom et $\mathrm{al}^{44}$ demonstrated the role of maternal responsiveness in atypical developmental patterns and increased temperamental difficulties in infants of mothers with PND at 48 months postpartum. They also found that full IQ scores were lower in children of mothers with PND, demonstrating the lasting effects of PND occurring early in the postpartum period. Recent systematic reviews by Kingston et $\mathrm{al}^{45}$ and Kingston and Tough ${ }^{46}$ evaluated longitudinal research of the effects of maternal distress, including postnatal distress, on infants and school-aged children. They reported particular effects of postnatal maternal distress on both infant ${ }^{45}$ and school-aged child ${ }^{46}$ cognitive and socio-emotional development. They also summarized small-to-moderate effects of postnatal maternal distress on the behavior of school-aged children. ${ }^{46}$

\section{The mother-infant relationship}

Developmental literature has highlighted the importance of early influence at protecting and promoting development. The infant-caregiver relationship has been widely recognized to play an important role in child development. ${ }^{47}$ Murray and Cooper ${ }^{39,40}$ suggested that the effects of PND on infant development were mediated through an association with maternal cognitions and maladaptive parenting practices. Research by Stein et $\mathrm{al}^{48}$ demonstrated that disturbances in maternal cognitions of women with PND may play a causal role in the negative effects on the mother-infant relationship.

Parental ability to regulate an infant's emotional state plays a key role in helping children to develop strategies for selfregulation. ${ }^{22,49-51}$ Gerhardt $^{50}$ summarized that the implications of failure of the caregiver to respond appropriately or "good enough" to her infant's needs had an impact at the neurochemical level of prolonged increase of cortisol levels on the infant. Gerhardt ${ }^{50}$ reviewed evidence that prolonged levels of cortisol in early infancy have consequences for neural systems implicated in how infants tolerate stress later in life, namely the prefrontal cortex and Hypothalamic-pituitary-adrenal axis.

Emerging imaging research completed with adult children of women with PND captured a significant association between their attachment security at 18 months and neural responding at 22 years of age. ${ }^{52}$ Specifically, the research found increased activation in prefrontal areas and decrease in activation of neural regulation of positive affect. Further research identified that compared with controls, women with PND are less able to identify happy faces potentially leading to decreased responsiveness toward their infants. ${ }^{53}$

Van Den Boom ${ }^{54}$ reported that educating vulnerable parents on how to respond appropriately and "optimally" to their temperamentally reactive infants was central to forming secure attachment bonds with their infants. This secure attachment, which develops between the mother and infant, also illustrates that the care the infant receives can impact in a protective manner on the developing child. Consequently, it seems that at least optimal parenting is a key feature in a parent's (namely the mother's) ability to regulate and soothe his/her infant during periods of distress. ${ }^{50}$

\section{Interventions focusing on the mother-infant relationship}

There is a body of research investigating various treatment approaches for PND. Within the available literature, several approaches have been identified and have demonstrated variable levels of efficacy, including various antidepressant treatments, ${ }^{55,56}$ antenatal group interventions, ${ }^{57}$ psychoeducation, ${ }^{58,59}$ cognitive behavior therapy (CBT), ${ }^{60,61}$ interpersonal psychotherapy (IPT), ${ }^{34,62,63}$ and interventions focusing on the mother-infant relationship ${ }^{64-66}$ and baby massage. ${ }^{67,68}$ Indeed, there are several comprehensive literature reviews on the evidence base of different interventions. ${ }^{12,69-73}$

However, within this literature, a poverty of interventions measuring outcomes relating to the mother, her relationship with the infant, and infant development has been identified. The majority of reviews on the subject have explored efficacy in relation to maternal mood. Furthermore, there is emerging evidence that the treatment of PND alone is not sufficient to improve the mother-infant relationship as well as child development. ${ }^{34,69}$ Given that PND affects the mother, her relationship with the infant, as well as the infant's development and well-being, a systematic review exploring the impact of interventions on these outcomes was clearly indicated.

\section{Assessment of child outcomes and dyadic relationship in interventions for PND}

A significant proportion of PND treatment literature has focused on the mother's depression in isolation, with few studies assessing the quality of the dyadic relationship as well as child developmental outcomes. They do not reflect mechanisms or moderators proposed by Goodman and Gotlib. ${ }^{37}$ Therefore, it is difficult to determine what impact treatment has beyond outcomes associated with maternal mood.

While there is an extensive literature of evaluation studies on various interventions for PND, little is known about the benefit of interventions to the quality of the mother-infant relationship and moreover, child developmental outcomes. Poobalan et $\mathrm{al}^{74}$ addressed this issue through an earlier review 
of treatments for PND, which focused on the mother-infant dyad relationship. Outcomes were discussed in terms of child outcomes. They noted some support for dyadic-focused interventions in improving child outcomes; however, the evidence was equivocal. In addition, Poobalan et al's review ${ }^{74}$ did not calculate effect sizes. In contrast, the present review extends the review by Poobalan et $\mathrm{al}^{74}$ by reporting on effect sizes, updating the search period from 1999 to 2014, inclusion of other therapies (antidepressant medication), and rigorous quality assessment, using the Clinical Tool for Assessment of Methodology (CTAM $)^{75}$ categories including allocation, assessment, control groups, analysis, and treatment. Most importantly, the present review considers the impact of treatments on maternal depression symptoms in addition to child outcomes.

The aims of the present systematic review were to evaluate all trials reported in the literature since 1999 and to evaluate intervention research, which has included outcomes measuring the quality of the mother-infant relationship and/or child developmental outcomes in addition to maternal mood.

\section{Method}

\section{Search strategy}

The literature search included publications from 1999 to 2014, since an earlier review by Poobalan et $\mathrm{al}^{74}$ reviewed studies from the 1990 s to 2005 , using a standard assessment adapted from the Cochrane Collaboration and Jadad Scale. ${ }^{76}$ The following databases were searched: PsycINFO, Medline, Embase, Web of Science, and Maternity and Infant Care. Additional searches were run using the aforementioned databases and PubMed for the dates between 2012 and 2014. Boolean searches on MeSH were conducted using combinations of the following (and related) terms: (“depression, postpartum" [MeSH Terms] OR "depression, postpartum" [MeSH Terms]) AND (("therapy" [Subheading] OR "therapy" [All Fields] OR "treatment" [All Fields] OR “therapeutics" [MeSH Terms] OR "therapeutics" [All Fields]) OR ("Intervention (Amstelveen)" [Journal] OR “intervention" [All Fields] OR "Interv Sch Clin" [Journal] OR “intervention" [All Fields])) AND (("mother-child relations" [MeSH Terms] OR ("mother-child" [All Fields] AND "relations" [All Fields]) OR "mother-child relations" [All Fields] OR ("mother" [All Fields] AND "child" [All Fields] AND "relations" [All Fields]) OR "mother child relations" [All Fields]) OR ("child development" [MeSH Terms] OR ("child" [All Fields] AND "development" [All Fields]) OR "child development" [All Fields] OR ("infant" [All Fields] AND “development" [All Fields]) OR "infant development"
[All Fields])) AND (“1999/01/01” [PDAT]: “2014/12/31” [PDAT]), Filters: Journal Article, From January 1, 2012 to December 31, 2015, Humans). All titles and abstracts were initially scanned for relevance.

\section{Inclusion criteria}

Studies were considered if they included a treatment or intervention which was delivered in the postnatal period, and if the primary outcomes assessed maternal depression and mother-infant interaction and/or child outcomes. Both single-group and randomized controlled trial (RCT) designs were considered for inclusion. A further inclusion criterion was that participants were experiencing low mood as indicated by a screening tool (ie, Edinburgh Postnatal Depression Scale [EPDS]) or a professional diagnosis of depression.

\section{Exclusion criteria}

Studies were excluded if they were single-case designs, reviews, book chapters, and/or discussion papers, not in the English language, and not peer reviewed.

\section{Evaluation of quality of trial methodology}

The CTAM, an assessment tool used to evaluate the quality of psychotherapeutic trials, ${ }^{75}$ was used in the present study because of its comprehensiveness in covering the six main areas of trial design, including sample size and recruitment method, allocation to treatment, assessment of outcomes, control groups, description of interventions, and analysis of data. There are a total of 15 items. Scores range from 0 to 100 ; scores over 65 are regarded as good quality.

Effect sizes indicate the magnitude of difference between two groups. In this review, they were also calculated separately for maternal mood, quality of dyadic relationship, and child developmental outcomes.

Effect sizes (Cohen's $d$ ) were considered as small if between 0.2 and 0.3 , medium if between 0.4 and 0.7 , and as large if equal to or greater than 0.8 . As suggested by Cohen, ${ }^{77}$ effect sizes were calculated individually given the heterogeneity of outcome measures and interventions. Effect sizes have only been calculated in studies where means and standard deviations were reported. Effect sizes have not been calculated in previous reviews of this literature.

\section{Results}

The initial search returned 862 articles. Six hundred and seventy-two articles did not meet inclusion criteria on the 
basis of a review of the title and/or abstract. A further 143 articles were excluded after more detailed examination of the title and abstract. Twenty-eight articles were removed for being duplicates or triplicates leaving 19 studies to be evaluated (Figure 1) for a schematic diagram of the literature search. Only three studies ${ }^{34,61,64}$ assessed both the quality of the dyad relationship and child outcomes. Although there was a high degree of heterogeneity across studies and measures used for assessment, effect sizes for different outcomes (maternal mood, mother-infant relationship, child developmental outcomes) were calculated where possible.

\section{Location and sample}

From the 19 studies included in the review, nine were carried out in the USA, five in the UK, two in the Netherlands, two in Australia, and one in Canada.

\section{Participant characteristics}

Of the 19 studies, ten were carried out with a multiparous sample and three with a primiparous sample, and six of the studies did not report parity. There was high variability across study client inclusion criteria regarding how depressive diagnosis was determined. Thirteen studies included participants with a professional diagnosis of PND, and six

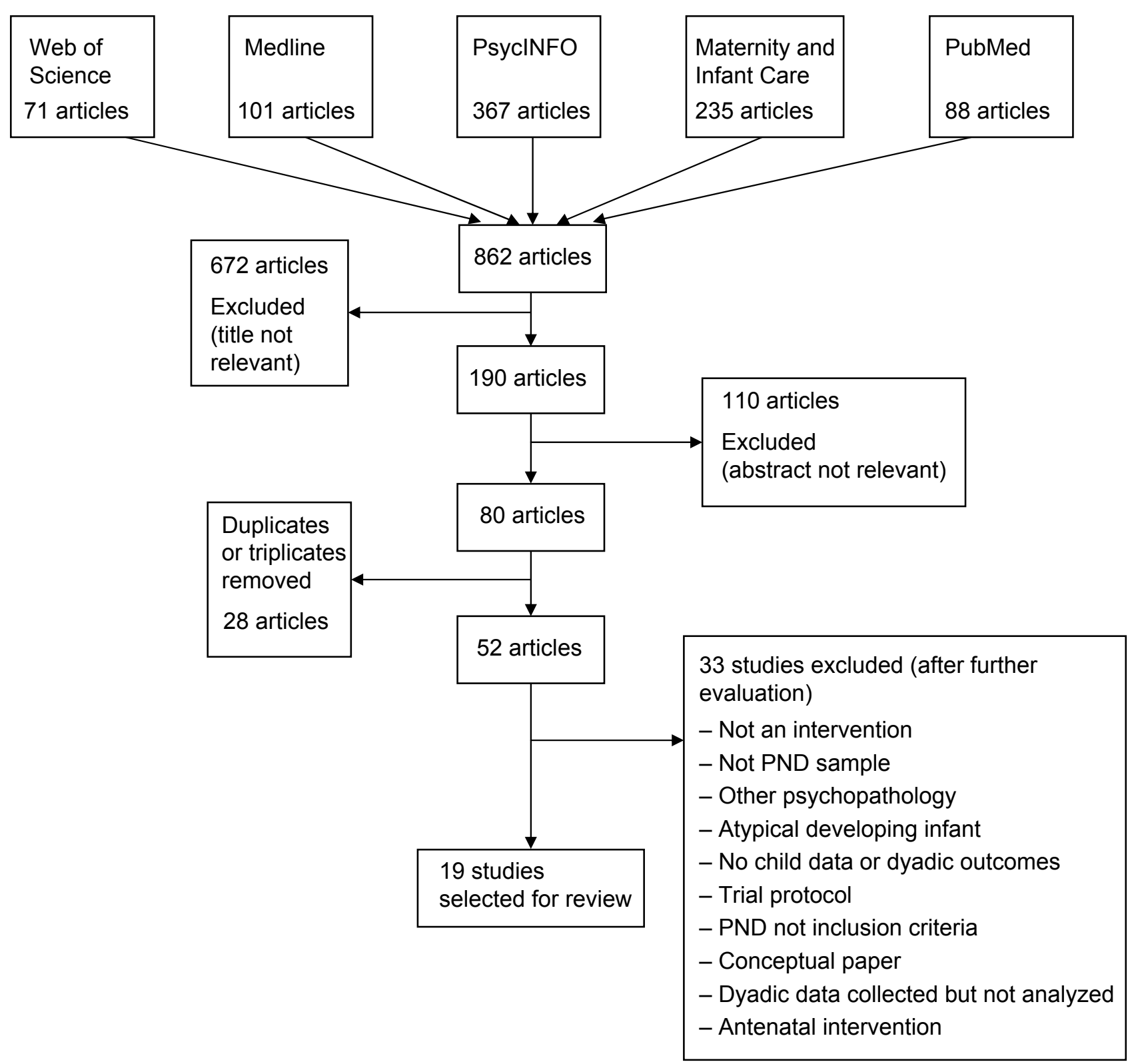

Figure I Schematic diagram of literature search for studies on treatment for PND with outcomes assessing mother-infant interaction and/or child outcomes. Abbreviation: PND, postnatal depression. 
studies included participants with probable diagnosis through public health screening. There were also differences across characteristics of participants in terms of severity of depression, marital status, and age of baby (Table 1).

\section{Treatment type, session length, and total duration}

The types of interventions evaluated in this review varied greatly with respect to their focus. For example, some interventions focused on the dyadic interaction, whereas others focused on maternal depression. Clark et $\mathrm{al}^{65,78}$ evaluated mother-infant therapy group (M-ITG), a relationship-focused intervention grounded in interpersonal, psychodynamic, and family system approaches. The M-ITG intervention focuses on a) providing therapeutic intervention and peer support, b) addressing infant emotional regulation and social engagement, and c) promoting sensitive interaction in the dyad. In their 2003 study, Clark et al ${ }^{65}$ compared M-ITG with IPT, which involves identifying interpersonal patterns contributing to symptoms of PND.
Forman et $\mathrm{al}^{34}$ and Mulcahy et $\mathrm{al}^{62}$ also examined IPT and described it as focusing on social role transitions (ie, transition to parenthood) as well as loss and grief in addition to focusing on individual interpersonal aspects. Infant massage, which involves administering various massage techniques to an infant's body while adjusting strokes according to infant responses, was also evaluated. ${ }^{67,68,79}$

A further intervention focusing on the dyad relationship, called interaction coaching intervention, was evaluated by Horowitz et al. ${ }^{80}$ The intervention is designed to strengthen the dyadic relationship and focuses on a) promoting maternal responsiveness, b) guiding mother to make eye contact with infant, c) responding to pauses (ie, imitation, facial expression, and tone), d) practicing through trial and error, e) reinforcing maternal-sensitive responsiveness, and f) praising success.

Kersten-Alvarez et $\mathrm{al}^{81}$ also evaluated a mother-infant intervention focusing on enhancing quality of dyadic interaction, through improving maternal sensitivity using video feedback and where needed, using modeling behavior,

Table I Participant characteristics including marital status, age of baby and mother, and level of depression (at baseline) across all studies

\begin{tabular}{|c|c|c|c|c|}
\hline Study & Marital status & Age of baby & $\begin{array}{l}\text { Level of depression } \\
\text { (baseline) }\end{array}$ & Mean age of mother \\
\hline Field et $\mathrm{al}^{79}$ & Single parents only & I-3 months (range) & Not reported & 17.3 years $($ range $=\mid 4-19)$ \\
\hline Horowitz et $\mathrm{al}^{80}$ & Not reported & 4-18 weeks (range) & I 4.4 (mean BDI) & $3 \mid$ years $($ range $=|7-4|)$ \\
\hline Onozawa et al ${ }^{68}$ & $\begin{array}{l}91 \% \text { married/ } \\
\text { cohabiting }\end{array}$ & $\begin{array}{l}\text { 8.6-9.0 months } \\
\text { (median range) }\end{array}$ & $\begin{array}{l}\text { Baseline EPDS } \\
\text { (median scores) }\end{array}$ & I8-45 years (range) \\
\hline Clark et $\mathrm{al}^{18}$ & $84.6 \%$ married/cohabiting & 8.9 months (range I-24 months) & $>16(\mathrm{BDI})$ & 31.4 years $($ range $=19-44)$ \\
\hline $\begin{array}{l}* \text { Murray et al }{ }^{61} \\
* \text { Cooper et } \mathrm{a}^{82}\end{array}$ & $88 \%$ married/cohabiting & 8 weeks & $>12$ (EPDS) & 27.7 years (range $=17-42$ ) \\
\hline Milgrom et al ${ }^{60}$ & $79.6 \%$ with partner & 18.13 weeks (mean) & I7.0 (mean BDI) & 29.9 years \\
\hline Jung et $\mathrm{al}^{66}$ & $100 \%$ married/cohabiting & 3.5 months (range $=3-4$ months) & $>10$ (BDI/EPDS) & 33 years $($ range $=2|-4|)$ \\
\hline Forman et $\mathrm{al}^{34}$ & $100 \%$ married/cohabiting & 6.1 months (mean) & Not reported & 30.6 years \\
\hline Clark et $\mathrm{al}^{65}$ & Not reported & $\begin{array}{l}6.4 \text { months } \\
\text { (range }=1.00-24.26 \text { months) }\end{array}$ & $\begin{array}{l}22.3 \\
\text { (mean BDI) }\end{array}$ & 31.3 years \\
\hline O'Higgins et $\mathrm{al}^{67}$ & $87 \%$ married/cohabiting & 19 weeks & I 3.5 (mean EPDS) & Not reported \\
\hline **Van Doesum et al ${ }^{64}$ & $91.3 \%$ cohabiting & 5.5 months (mean) & 23.9 (mean BDI) & 30.1 years \\
\hline Logsdon et $\mathrm{a}^{55}$ & $\begin{array}{l}55.5 \% \text { single } \\
40.7 \% \text { married } \\
3.7 \% \text { divorced } / \text { separated }\end{array}$ & 10 months (mean) & I8.I (mean HRSD) & 24.5 years \\
\hline $\begin{array}{l}* * \text { Kersten-Alvarez } \\
\text { et a }\left.\right|^{81}\end{array}$ & $\begin{array}{l}85 \% \text { married/cohabiting } \\
16 \% \text { single }\end{array}$ & 6 months (mean) & 23.8 (mean) & 35.7 years (range $=25-43)$ \\
\hline Beeber et $\mathrm{a}^{83}$ & $37 \%$ cohabiting & 24.9 months (mean) & I6.2 (HRSD) & 26 years \\
\hline Horowitz et al ${ }^{84}$ & $83 \%$ cohabiting & 7.4 weeks (mean) & I2.34 (EPDS) & 31 years \\
\hline Tsivos et al ${ }^{86}$ & $\begin{array}{l}89 \% \text { married/cohabiting } \\
\text { II\% separated/divorced }\end{array}$ & 6.2 months (mean) & 32.4 (BDI-II) & 28.4 years \\
\hline Goodman et al ${ }^{85}$ & $\begin{array}{l}59.5 \% \text { married/cohabiting } \\
16.6 \% \text { single }\end{array}$ & 6 weeks & I2.3 (EPDS) & 30.7 years \\
\hline
\end{tabular}

Notes: *Cooper et a ${ }^{82}$ report the maternal mood data, and Murray et al ${ }^{\mid 61}$ report dyad and child outcome. ${ }^{* *}$ Kersten-Alvarez et al ${ }^{81}$ report the dyad and child developmental outcomes of Van Doesum et al. ${ }^{64}$

Abbreviations: BDI, Beck Depression Inventory; EPDS, Edinburgh Postnatal Depression Scale; HRSD, Hamilton Rating Scale for Depression. 
cognitive restructuring, support, and baby massage. Jung et $\mathrm{l}^{66}$ investigated the efficacy of a further dyadic-focused intervention. Keys to Caregiving, aims to facilitate sensitive responding to infant behaviors through enhancing understanding of the meaning of different infant behaviors. The intervention involved practice with the infant during the session and at home. Keys to (caregiving) Capital sessions included understanding of infant states, infant behaviors, infant cues, modulation, and feeding.

In their RCT, Cooper et $\mathrm{l}^{82}$ compared routine care (health visiting with no additional input) with $\mathrm{CBT}$, nondirective counseling, and psychodynamic therapy. Nondirective counseling provided women with the opportunity to discuss feelings of current concerns (ie, financial, relational - partner/ infant). CBT was tailored toward the mother's management of her infant (ie, feeding, sleep) and the quality of the dyadic interaction. Within the CBT sessions, a woman was also encouraged to problem solve in a systematic way, and examine patterns of thinking about her infant and herself as mother. Psychodynamic therapy utilizes techniques aimed at understanding mother's representation of infant and relationship with infant promoted by exploring aspects of mother's early attachment history. Two antidepressant medications (nortriptyline and sertraline) were also evaluated. ${ }^{55}$

The review of all studies also showed that session lengths ranged from 15 minutes to 2 hours, and total treatment duration ranged from three to 12 sessions. Mode of delivery included both individual and group delivery as well as mixed individual and group. A summary of type of treatment, session length, and treatment duration, CTAM scores, and domains of assessment (maternal affect, dyad relationship, and child development) across all studies is presented in Table 2 .

\section{Methodological quality}

A summary of CTAM scores across all studies assessed is presented in Table 2. Overall, most studies (eleven of the 19 studies) included in the review had a CTAM score below 65, which is described as inadequate by the authors of the CTAM. $^{75}$

\section{Sample}

Most studies (12/19) used a convenience sample. Nine of the 19 studies had a sample size greater than 27 in each treatment group. ${ }^{34,61,64,67,80-84}$ Numbers of less than 27 in each group are regarded as inadequate and do not score on the CTAM. Small sample sizes are a long-standing limitation within the PND literature. A large proportion of studies with PND populations failed to recruit to target, and as such they were underpowered. This is a difficulty experienced across trials.

\section{Allocation}

While most studies described whether there was true random allocation or minimization allocation across treatment groups, only ten studies described the process of randomization. ${ }^{55,61,62,64,67,80,82,83,85,86}$ Furthermore, four studies also indicated that the process of randomization was carried out independently of the research team. ${ }^{55,62,83,85}$

\section{Assessment}

All of the studies used standardized assessments to measure outcomes. All but three studies ${ }^{66,68,86}$ had assessors who were independent of treatment delivery (ie, they were not the therapist on the trial). Eight studies reported that assessments were carried out blind to treatment group allocation. . $^{34,61,67,80-83,85}$ However, only two studies ${ }^{68,80}$ described the method of rater blinding, while only one study ${ }^{68}$ reported verification of rater blinding.

\section{Control groups}

While most studies utilized a RCT design, three studies ${ }^{34,67,83}$ reported using both, no treatment or waitlist control (WLC) group and a control group that controlled for nonspecific effects (ie, nondepressed comparison group).

\section{Analysis}

All studies conducted appropriate analyses given their design and sample sizes. One study ${ }^{34}$ employed an intention-to-treat analysis (including all participants as randomized), and six studies had attrition of less than $15 \%{ }^{61,62,80,82,84,85}$ The remaining studies did not handle drop-outs appropriately, and had attrition of greater than $15 \%$ or inappropriate sample sizes (ie, less than 27 participants in each group).

\section{Active treatment}

All interventions employed were psychotherapy or psychosocial interventions with the exception of one study, ${ }^{55}$ which was an evaluation of two types of antidepressant medications. Nine of the studies provided an adequate description of the treatment, reported the use of a protocol or manual, as well as an assessment of adherence to the protocol. ${ }^{62,64,65,78,81-83,85,86}$

\section{Maternal mood, dyadic relationship, and developmental outcomes \\ Outcome measures}

Various outcome measures were used across the studies to evaluate the efficacy of interventions in the domains of 


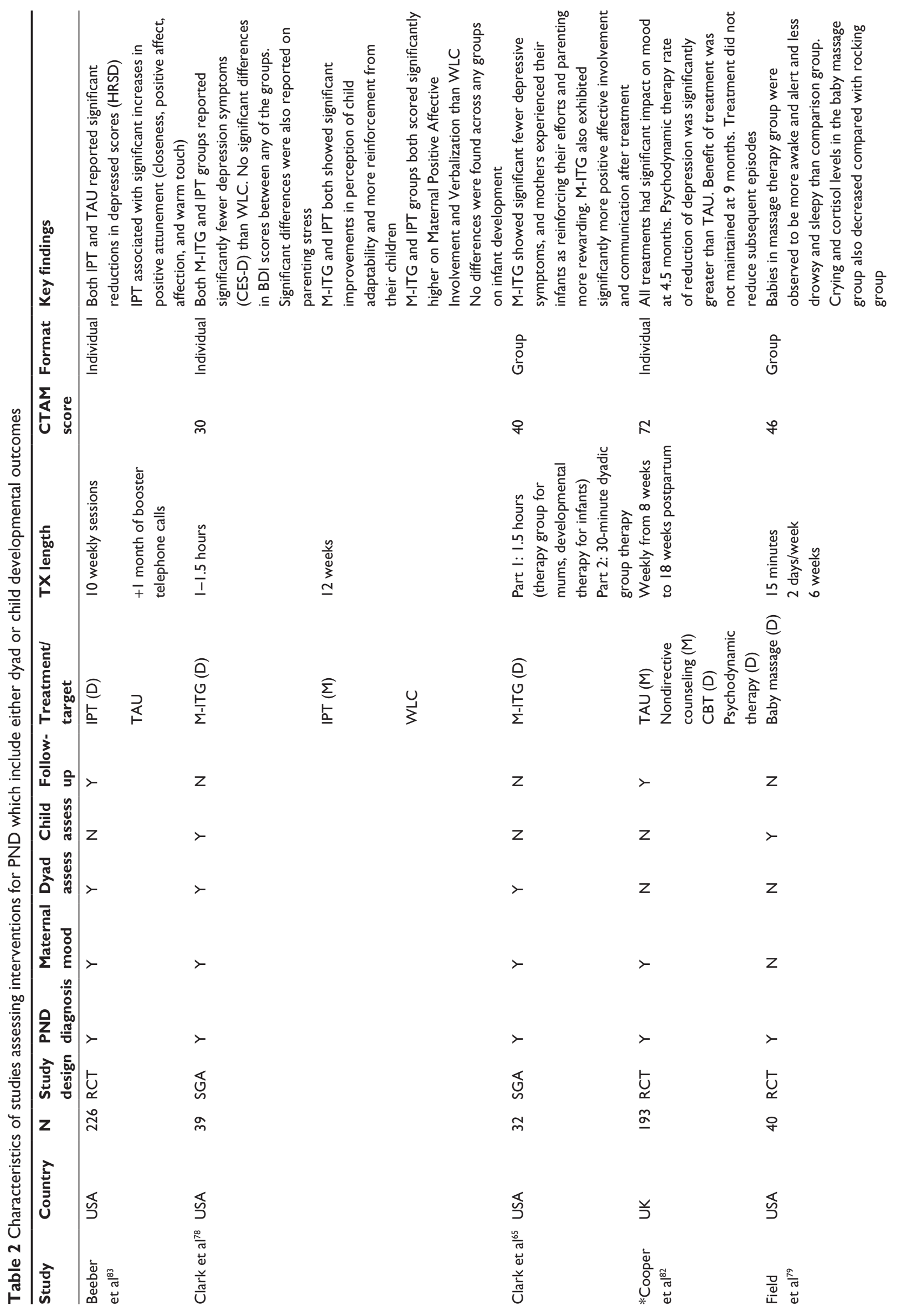




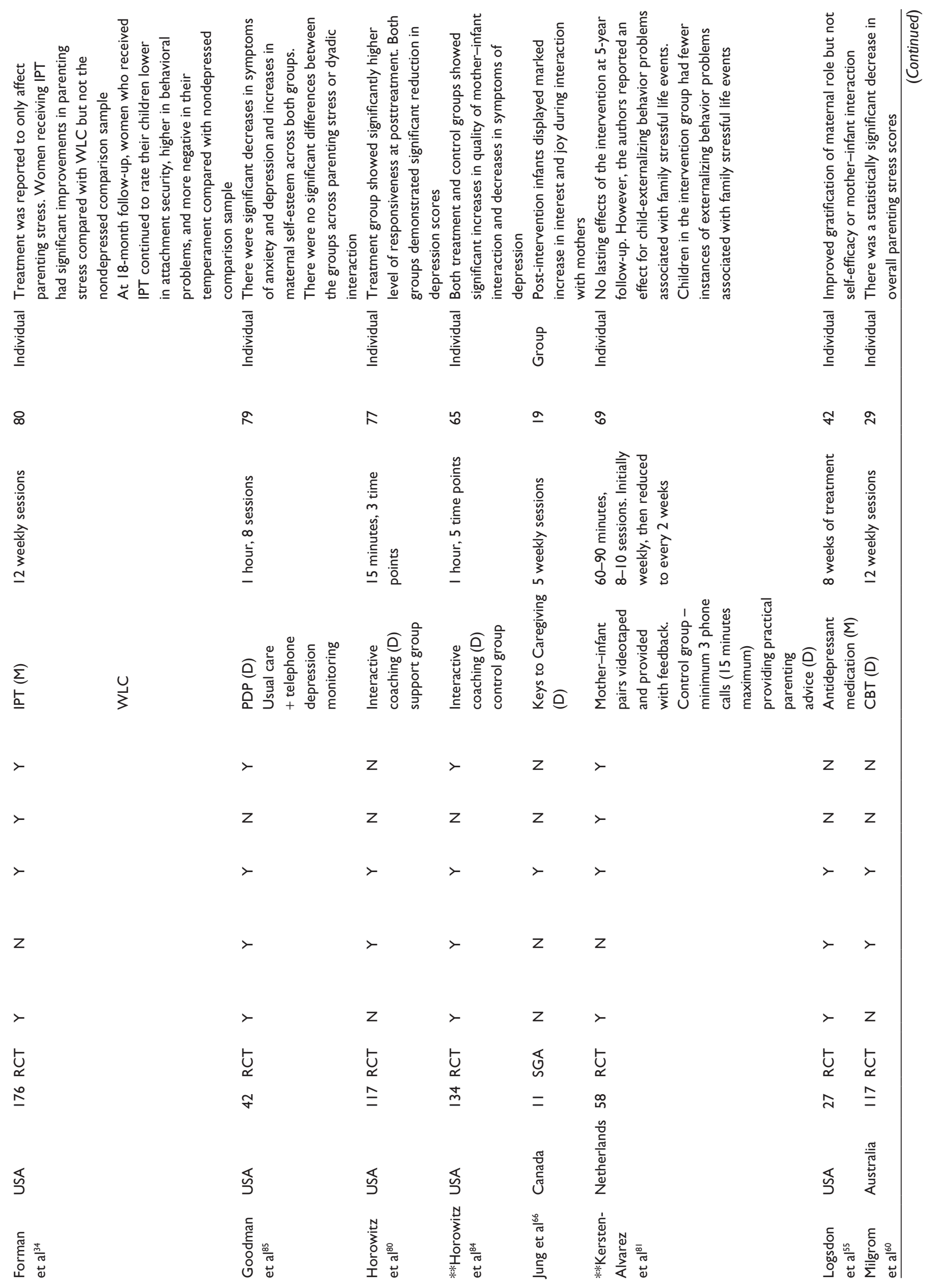




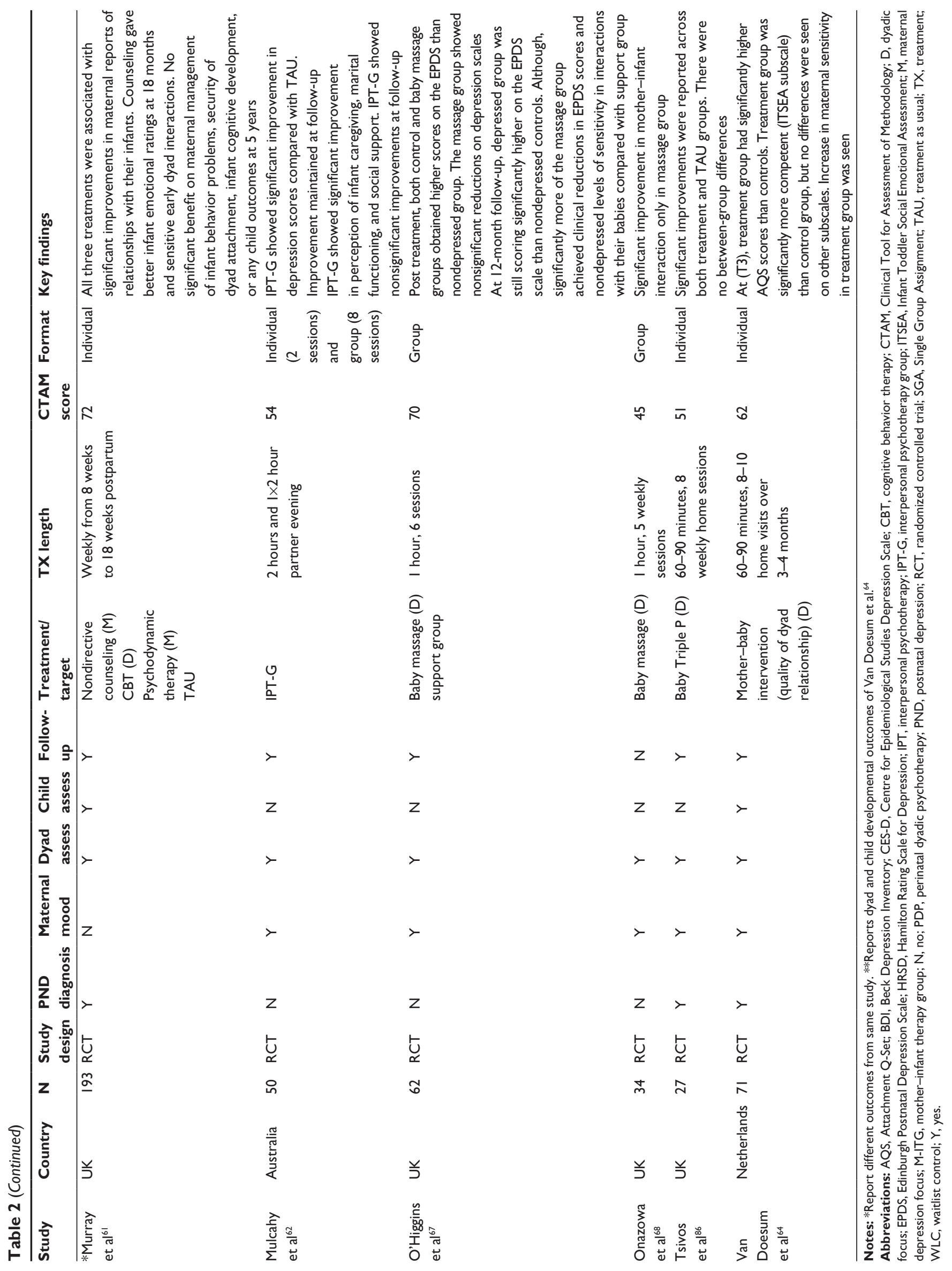


maternal affect, dyad relationship, and child development (Table 2).

\section{Maternal mood}

Studies used either the Beck Depression Inventory or the EPDS to assess maternal mood, with the exception of three studies, which used the Hamilton Rating Depression Scale..$^{55,62,83}$ The largest effect sizes, though moderate in terms of effectiveness of treatment on improvement in maternal mood, were reported by Horowitz et $\mathrm{al}^{80}$ and Clark et $\mathrm{al}^{65}$ (Table 3 presents the effect sizes). In their evaluation of the efficacy of a behavioral intervention delivered by advanced practice nurses and research assistants, which involved coaching designed to promote maternal responsiveness, Horowitz et $\mathrm{al}^{80}$ reported that women who had received the behavioral coaching showed a significantly higher level of responsiveness posttreatment. Clark et al ${ }^{65}$ investigated the efficacy of a 12-week, manualized M-ITG compared with WLC: women allocated to the M-ITG showed significantly fewer depressive symptoms, and experienced their infants as more reinforcing and parenting more rewarding.

Smaller effect sizes (Table 3) were calculated across several other studies. ${ }^{62,64,67,78,82}$ Three studies reported on evaluations of IPT: Mulcahy et $\mathrm{al}^{62}$ in an RCT comparing group IPT with treatment as usual (TAU), Clark et $\mathrm{al}^{65}$ in an RCT comparing M-ITG and IPT with WLC, and Beeber et $\mathrm{al}^{83}$ in an RCT comparing IPT + parental enhancement (PE) with an attention control health education condition. While a greater effect size was calculated for M-ITG compared to IPT in Clark et al's ${ }^{65}$ study, an overall greater effect size was reported by Mulcahy et al. ${ }^{62}$

Cooper et $\mathrm{al}^{82}$ compared counseling, psychodynamic therapy, and CBT with TAU. The largest effect size was calculated for psychodynamic therapy. Psychodynamic therapy was associated with significantly greater reductions in depressive symptoms compared with TAU; however, the effects were not maintained at 9-month follow-up. No significant differences were found in symptoms of depression between women who took part in an intervention working on the quality of the dyadic relationship compared with mothers receiving telephone parenting support only. ${ }^{64}$

Goodman et $\mathrm{al}^{85}$ evaluated the efficacy of perinatal dyadic psychotherapy (PDP), designed to reduce symptoms of depression and improve the mother-infant relationship. Using an RCT design, they compared PDP with a control condition receiving telephone monitoring of symptoms. Both groups reported significant improvements in their depressive symptoms.
Three studies evaluated different delivery modalities of baby massage. ${ }^{67,68,79}$ No significant differences were found across measures of maternal mood between comparison groups and women receiving baby massage in the studies by O'Higgins et al. ${ }^{67}$ Onozawa et $\mathrm{al}^{68}$ and Field et $\mathrm{al}^{79}$ did measure developmental but not maternal outcomes (but included women on the basis of formal diagnoses of major depression).

CBT supplemented with a mother-infant module Happiness Understanding, Giving and Sharing (HUGS) was evaluated by Milgrom et al. ${ }^{60}$ The authors reported significant reductions in symptoms of depression following CBT and further significant drops in parenting stress following the mother-infant module. Due to missing information, effect sizes for this study could not be calculated. Logsdon et $\mathrm{al}^{55}$ also reported significant reductions in symptoms of depression following 8 weeks of antidepressant treatment (nortriptyline and sertraline).

\section{Mother-infant relationship}

Of the 19 studies, 17 assessed dyadic relationship outcomes (Table 2). The measures used to assess the dyadic relationship varied widely. The largest effect size on dyadic outcome was calculated for the study by Kersten-Alvarez et al. ${ }^{81}$ The intervention (video feedback) had a medium effect on index of the quality of interactive behavior.

Clark et al' study ${ }^{78}$ had the second largest effect size calculated in the group that received IPT but only for factor one (Maternal Positive Affect Involvement and Verbalization) of the Parent-Child Early Relational Assessment (PCERA). ${ }^{87}$ A later study carried out by Clark et al ${ }^{65}$ found comparable findings using the PCERA, with the largest effect size calculated for factor one (as above), followed by factor two (Maternal Negative Affect and Behavior), six (Infant Dysregulation and Irritability), and seven (Dyadic Mutuality and Reciprocity).

Beeber et $\mathrm{al}^{83}$ also reported significant outcomes in their investigation of IPT + PE. They reported significant increases in positive involvement in the mothers who received IPT + PE only.

In their uncontrolled study, Jung et $\mathrm{al}^{66}$ reported that postintervention, infants displayed a marked increase in interest and joy during interaction with their mothers.

In another RCT, mother-infant dyads were either videotaped and given feedback using one of four techniques during eight to ten sessions, including (1) modeling, (2) cognitive restructuring, (3) practical support, and (4) baby massage, or provided with three sessions of practical parenting advice via telephone calls. ${ }^{64}$ The authors reported that at 6-month 


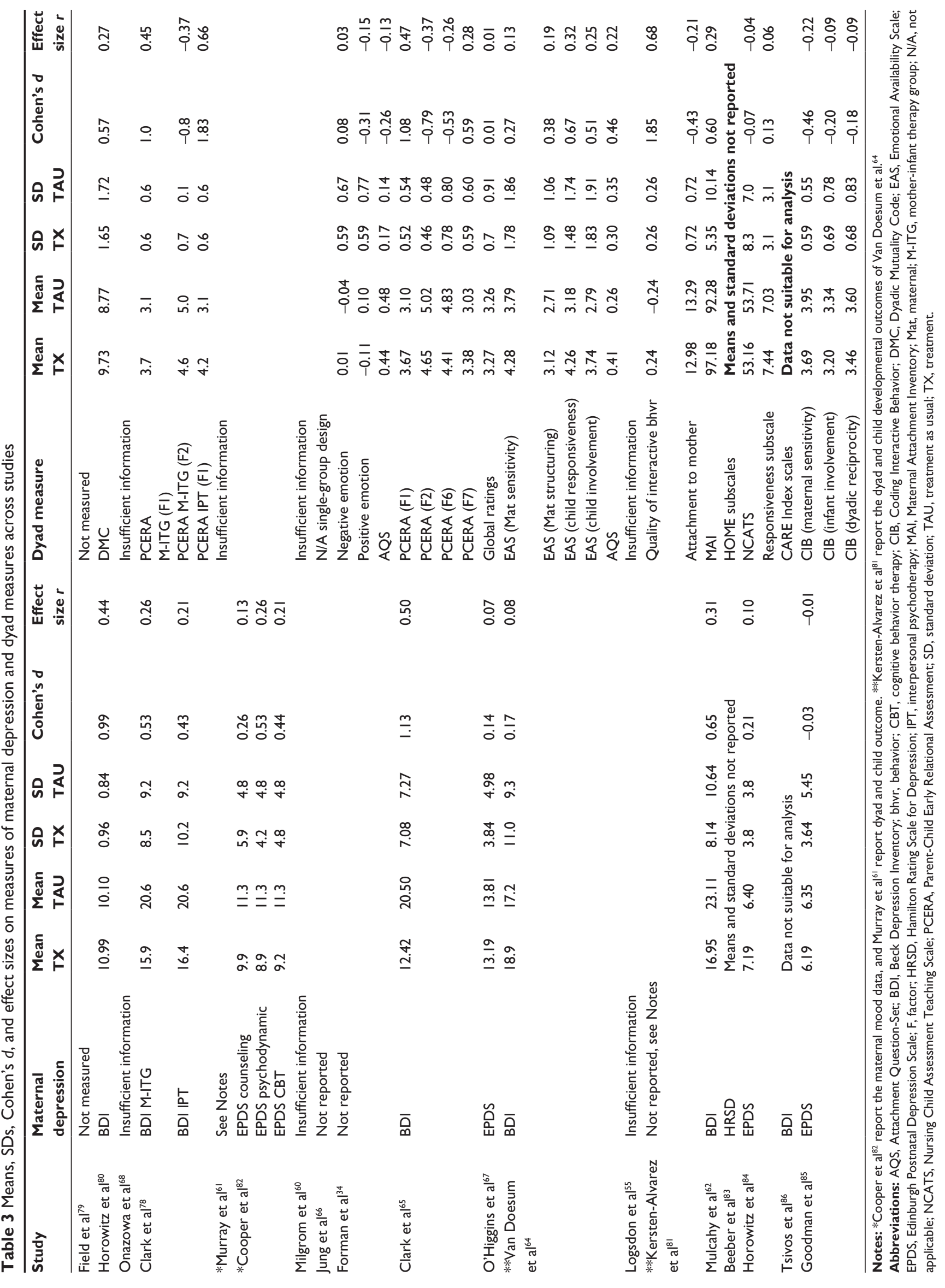


follow-up, the treatment group had higher Attachment Q-Set (AQS) scores and maternal sensitivity (on the Emotional Availability Scale [EAS] subscale) than controls. Small effect sizes were calculated for the video feedback intervention on child responsiveness and involvement, as well as maternal structuring and sensitivity EAS subscales. A small effect size was also calculated for the intervention on the AQS. A small effect of IPT on the Maternal Attachment Inventory was also found in Mulcahy et al's ${ }^{62} \mathrm{RCT}$ investigating the effectiveness of group IPT.

In the study by Horowitz et $\mathrm{al}^{80}$ the relationship-focused behavioral coaching invention (CARE) was found to have a small effect on the quality of the mother-infant relationship, as measured by the Dyadic Mutuality Code. In a recent RCT of the CARE intervention, despite significant reductions in both symptoms of depression and improvements to the mother-infant relationship, there were no significant differences between women receiving the CARE intervention and those allocated to a control group. ${ }^{84}$

In an RCT examining the effectiveness of baby massage in the treatment of PND, no differences in the quality of the mother-infant relationship (measured by global ratings) were found between women receiving baby massage and those receiving support only at posttreatment. ${ }^{67}$ However, at 1-year follow-up, depressed dyads who had participated in baby massage had comparable scores of maternal sensitivity with nondepressed dyads, whereas women who had received only support performed significantly less well.

In a double-blind RCT of two antidepressants (nortriptyline and sertraline), the authors reported no significant differences in the improvement in the quality of the dyadic interaction on the Child and Caregiver Mutual Regulation Coding Scale. ${ }^{55}$

With respect to the remaining studies, it was not possible to calculate the effect size of interventions on the motherinfant relationship. In another trial of baby massage, the authors reported significant improvements in mother-infant interaction (assessed by global ratings for mother-infant interactions) in women who received baby massage compared with women who attended a support group only. ${ }^{68}$

In their RCT, Murray et $\mathrm{al}^{61}$ reported limited short- and long-term improvements in the mother-infant interactive quality following treatment in nondirective counseling, CBT, psychodynamic therapy, or TAU. They reported improvements across all groups in face-to-face mother-infant interactions but no significant differences between groups. However, they did report that women allocated to the control group had higher levels of maternal sensitivity at baseline compared with the other groups. Interestingly, they also reported that women with high levels of social adversity who received counseling were found to have higher levels of maternal sensitivity. No other differences in treatment with respect to the quality of the mother-infant relationship were found.

In the study by Milgrom et $\mathrm{al}^{60}$ which investigated CBT and the adjunct mother-infant intervention, significant marked (self-reported) improvements in the function of mother-infant relationship following the mother-infant adjunct module were reported.

In a pilot RCT comparing Baby Positive Parenting Programme (Baby Triple P), in addition to TAU with TAU only, there were significant improvements in depressive symptoms. ${ }^{86}$ There was, however, no significant additive effect of Baby Triple $\mathrm{P}$ demonstrated across outcomes of maternal mood or dyadic outcomes, measured by the CARE index. ${ }^{86}$

Finally, Goodman et $\mathrm{al}^{85}$ found no differences in dyadicrelated outcomes between women allocated to PDP and those in a control group.

\section{Child development}

Four studies measured child developmental outcomes. ${ }^{34,61,64,79}$ Measures used to assess child development varied making it difficult to compare effect sizes between studies. Across studies, it was reported that infants improved on some subscales but not others (Table 4).

Van Doesum et $\mathrm{al}^{64}$ reported that infants in the treatment group (video feedback) were significantly more competent (measured by Infant Toddler Social Emotional Assessment [ITSEA] subscale scores) compared with infants in the control group.

In an RCT investigating the efficacy of IPT treatment in women with PND, a small effect size was calculated for IPT on Child Behaviour Checklist subscales of internalizing and externalizing. ${ }^{34}$ No differences in any of the other ITSEA subscales were found. Insufficient data meant that it was not possible to calculate effect sizes for the remaining studies. ${ }^{61,79}$

In their RCT comparing group baby massage with a rocking group, Field et $\mathrm{al}^{79}$ reported several outcomes related to infant behaviors, including Thoman's system of sleep recording, salivary cortisol, weight, formula intake, temperament ratings, and urine assays to measure hormones associated with stress. Babies in the massage group were observed to be significantly more awake and less drowsy compared with the rocking group. Crying and cortisol level in the baby massage group also decreased significantly compared with the rocking 
Table 4 Means, SDs, Cohen's d, and effect sizes on measures of child development across studies

\begin{tabular}{|c|c|c|c|c|c|c|c|}
\hline Study & Measure & Mean TX & Mean TAU & SD TX & SD TAU & Cohen's d & Effect size $r$ \\
\hline \multirow[t]{5}{*}{ Field et $\mathrm{al}^{79}$} & Emotionality & 12.2 & 13.0 & 4.0 & 5.0 & -0.12 & -0.08 \\
\hline & Sociability & 19.9 & 18.4 & 4.0 & 4.0 & 0.36 & 0.18 \\
\hline & Soothability & 18.5 & 15.6 & 4.0 & 5.0 & 0.64 & 0.31 \\
\hline & Serotonin & $1,427.9$ & $1,132.4$ & 779.0 & 517.0 & 0.45 & 0.22 \\
\hline & Cortisol & 656.4 & $1,016.8$ & 340.0 & 523.0 & -0.82 & -0.34 \\
\hline Murray et $\mathrm{al}^{61}$ & Insufficient information & & & & & & \\
\hline Forman et $\mathrm{al}^{34}$ (compared treated & CBC internalizing & 46.14 & 49.06 & 7.23 & 7.49 & -0.40 & -0.20 \\
\hline recovered with treated non-recovered) & CBC externalizing & 47.34 & 46.50 & 9.05 & 10.10 & 0.09 & 0.04 \\
\hline Van Doesum et $\mathrm{al}^{64}$ & ITSEA (competence) & 1.40 & 1.22 & 0.28 & 0.30 & 0.64 & 0.30 \\
\hline
\end{tabular}

Abbreviations: CBC, Child Behaviour Checklist; ITSEA, Infant Toddler Social Emotional Assessment; SD, standard deviation; TAU, treatment as usual; TX, treatment.

group. There were also significant changes in temperament. Babies in the massage group were observed to be significantly more sociable, more easily soothed, and less emotional compared with babies who were in the rocking group.

Several child developmental and behavioral outcomes were measured at three time points by Murray et al. ${ }^{61}$ There was no significant effect of treatment group on early management of infant behavior following treatment (4.5 months). At 18-month follow-up, there was a significant effect of counseling (after controlling for maternal age) on infant emotional and behavioral problems (measured by the Behavioural Screening Questionnaire) ${ }^{88}$ Five years later, a nonsignificant effect of CBT treatment was found on infant emotional and behavioral problems (measured by Rutter A2 Scale), but there were no differences across interventions in teacher-reported child behavioral difficulties (measured by Preschool Behavior Checklist). There were also no differences across the treatment groups in measures of cognitive development at 18-month (Mental Development Index of the Bayley scales) and 5-year (General Cognitive Index of McCarthey Scales) follow-ups.

\section{Discussion}

This is the first review to date to rigorously evaluate the methodological quality of studies using the CTAM, calculate effect sizes (where possible), and compare outcomes related to both maternal mood and child development and/or the dyadic relationship.

Of the interventions reviewed here, those which have focused on the dyad relationship, namely mother-infant therapy ${ }^{78}$ and a coaching intervention, designed to promote maternal responsiveness, ${ }^{80}$ had the greatest efficacy at reducing symptoms of PND (where data were available to calculate effect sizes). However, effect sizes of the aforementioned studies for improvements in the quality of the mother-infant relationship were more modest by comparison.
The intervention, which focused on the quality of the dyad relationship, demonstrated the largest effect size with respect to improvement in the quality of the mother-infant relationship. However, this finding was not consistent across all outcomes. While the majority (18/19) of studies measured mother-infant interaction outcomes, only four studies measured child developmental outcomes, and the resulting effect sizes were small. This highlights a limited therapeutic effect across child outcomes, despite overwhelming research evidencing the impact of PND on short- and long-term developmental patterns. In terms of intergenerational transmission of risk to children, it is difficult to determine whether reported improvements in mother-infant relationships were due to improvements in developmental outcomes or improvements in PND, or whether there is a bidirectional link. ${ }^{34,69,74}$

The findings from this review are consistent with those reported by Poobalan et al, ${ }^{74}$ an earlier review of eight RCTs aimed at treating PND through targeting the motherinfant relationship. For example, improvements in dyadic interaction do not necessarily lead to an improvement in child developmental outcomes. Interestingly, the size of the intervention effect on maternal outcomes was discrepant with those calculated on measures of infant development and the quality of the mother-infant interaction. These findings suggest that improvements in maternal mood may be necessary but not sufficient to improve additional dyadic and/or child developmental outcomes alone. In line with the mechanisms implicated in the intergenerational model of risk, ${ }^{37}$ there may be multiple mediating and moderating factors implicated in the transmission of psychopathological risk from mother to infant.

The findings from this review have implications for the psychological treatment of PND and future research. While certain interventions have been shown to be effective for treating PND, the benefits for child development and the quality of the dyadic relationship are less clear, as evidenced 
by the discrepancy between effect sizes for improvements in maternal mood and dyad and developmental outcomes. Indeed, an improvement in maternal mood did not necessitate an improvement in developmental outcomes ${ }^{35}$ as illustrated by the disparity in maternal, dyadic, and child outcomes reported in the present review. However, the incongruence between early outcomes may also be the result of a time delay between improvements in maternal mood and expectations from the infant resulting in the observed discrepancy. There may be a period of adjustment for the infant following improvements in maternal mood resulting in an observed dis-synchrony between the dyad..$^{33}$ In order to investigate this, long-term assessments of the mother-infant relationship are warranted.

The findings from the present review suggest that a developmental perspective into the conceptualization of how PND affects the mother and infant is needed. The role parents play in regulating their infants' emotional states may be a key element in improving treatment efficacy and promoting longterm effectiveness. ${ }^{89}$ There is a strong impetus for focusing on parenting skills and strategies as a medium for strengthening and protecting the mother-infant relationship, given the difficulties they experience with parenting. Despite significant reductions in depressive symptomatology, effect sizes were generally modest. While improvements to maternal mental health have been assessed, it remains important to also assess both short- and long-term benefits, to the mother's ability to respond sensitively to her infant.

\section{Quality of evidence}

The impact of PND interventions on child development requires further research because it remains difficult to draw conclusions from the research or compare studies as a result of study limitations. A large proportion of the studies obtained inadequate scores on the CTAM. Many of the studies were characterized by small, biased sample sizes, which increases reporting of false-negative findings and the rejection of potentially effective treatments. Many also failed to describe the allocation and randomization process, thereby reducing methodological rigor. Furthermore, while most studies employed the use of blind assessors, the process of blinding was not described. Although the majority of the studies used an RCT design, many did not address drop-out appropriately (ie, only analyzing treatment completers). Fewer than half of the included studies described intervention protocol and/or methods to ensure treatment fidelity; this highlights a limitation regarding quality assurance. The methodological observations are comparable with the extant literature. ${ }^{90,91}$
The correlation between direction of effect size and strength of methodological quality is comparable to previous studies using the CTAM for assessment of trial methodological quality, which may be explained by the standard of reporting or length constraints of the journal in which they were published. One method for improving the quality of reporting RCTs is to implicate the use of Consolidated Standards of Reporting Trials, which outlines the gold standard for conducting RCTs. Alternately, as suggested by Cuijpers et $\mathrm{al}^{70}$ quality assurance may be achieved by adherence to the Cochrane Handbook which outlines four criteria in ensuring quality assurance. Only four studies in this review presented Consolidated Standards of Reporting Trials or participant flow diagrams.

There were also several observations with regard to participant characteristics, which warrant attention. Firstly, there was wide heterogeneity across study client inclusion criteria. Specifically, some studies included clients with a diagnosis of PND made by a professional, while others relied on screening measures alone, such as the EPDS. Although probable diagnosis is cost- and time-effective, there is a limitation that researchers who rely exclusively on screening questionnaires for eligibility are including participants who are experiencing comorbid diagnoses, which may invariably influence treatment efficacy. Secondly, we observed homogeneity in marital status, with a large proportion of women being in married/ cohabiting relationships. Thirdly, there was a large degree of variability with regard to variables including age of the infant and severity and course of the depressive episode.

An additional observation is the impact that parity has on outcomes. For example, difficulties experienced may vary depending on whether mothers are primiparous or multiparous. Indeed, research has shown that primiparous and multiparous mothers experience different mother-infant relationship problems at 3 months postpartum. ${ }^{92}$ This variance may be explained by differences between multiparous and primiparous mothers in adjustment to parenthood. ${ }^{93}$

These methodological observations make it difficult to determine what to target in treatment, how long to do it for, and what delivery modality should be used. McLennan and Offord ${ }^{35}$ have suggested that further research is needed to establish the role of PND as a risk factor to determine whether it should be targeted for improving developmental outcomes.

\section{Recommendations for future research}

Future research should include developmental and predictive measures of vulnerability toward future developmental 
psychopathology because these measures provide an index of long-term effectiveness. ${ }^{94}$ There is also a need to acknowledge fathers when administering treatment. ${ }^{19}$ According to the Goodman and Gotlib model, ${ }^{37}$ fathers may moderate the transmission of risk..$^{95,96}$ Since it is a period of adjustment, fathers face challenges in becoming new parents, including redefining their relationship and roles with their spouse and importantly learning to respond adaptively to their babies. ${ }^{97}$ Research will need to consider the dyadic relationship and interactions between both parents and the developing infant. Risk factors (ie, sociodemographics) associated with PND as well as the concept of sensitive periods in development and resilience to adversity (infants are particularly vulnerable to PND, due in part to development of neuroregulatory mechanisms) need to be kept in mind..$^{22,37}$

Research with clients from underrepresented groups with PND, including black and ethnic minority populations, is also needed. We found no studies which investigated effectiveness of interventions for PND in low-income or developing nations' populations.

A further consideration for future research is the revisions to the diagnostic criteria which have altered the conceptualization of PND to include mood disorders with an onset in pregnancy. Sharma and Mazmanian ${ }^{98}$ highlighted that the inclusion of the prepartum and mixed feature specifiers would lead to increased awareness, monitoring, and appropriate timely treatment of women who are at risk of mood difficulties during their pregnancy, including mania, hypomania, and mixed episodes. However, they cautioned that the inclusion of the prepartum specifier may obscure etiological, clinical, and treatment differences between those with prepartum and postnatal onsets.

\section{Limitations}

The findings from this review are subject to some limitations. Firstly, strict search terms were used due to the volume of papers returned in initial searches. Hence, it is possible that some studies were excluded. Secondly, it was not possible to calculate effect sizes across all domains of assessment (maternal mood, mother-infant relationship, child developmental) due to missing data. Thirdly, there was a degree of variability regarding how a diagnosis of PND was established. For example, the inclusion of studies assessing participant eligibility through the use of screening measures (ie, the EPDS) and not formal diagnosis may have affected the reliability with which the results were interpreted.

Despite the evidence for the benefits, the review literature on the subject has highlighted that there is insufficient evidence to recommend a specific treatment, and therefore, further research is warranted. ${ }^{70,74,91}$

\section{Conclusion}

Maternal well-being and child development are inextricably linked. Our review has highlighted the poverty of interventions assessing outcomes relevant to both mother and infant. These findings highlight the need for further research to continue to measure the quality of the mother-infant relationship but also to add measurements of child development and longterm outcomes to their research programs. Further research addressing the highlighted methodological limitations is warranted. Until then, we can make no recommendation for any intervention in particular.

\section{Acknowledgment}

We would like to acknowledge Nadine Santos for her assistance in preparing the revised manuscript.

\section{Authors' contributions}

All authors contributed toward data analysis, drafting and revising the paper and agree to be accountable for all aspects of the work. ZLT conducted the systematic literature search and initial analysis. The first draft was prepared by ZLT and finalized by AW. All authors contributed to and have approved the final manuscript.

\section{Disclosure}

No author has any share or ownership in Triple P International Pty Ltd. Matthew Sanders is the founder and an author of various Triple $\mathrm{P}$ programs and a consultant to Triple $\mathrm{P}$ International Pty Ltd. The authors report no other conflicts of interest in this work.

\section{References}

1. Milgrom J, Martin PR, Negri LM. Treating Postnatal Depression: A Psychological Approach for Health Care Practitioners. London: Wiley; 1999.

2. Cooper P, Murray L. The impact of psychological treatments of postpartum depression on maternal mood and infant development. In: Murray LCPJ, editor. Postpartum Depression and Child Development. New York, NY: Guilford Press; 1997:201-220.

3. Coates A, Schaefer C, Alexander J. Detection of postpartum depression and anxiety in a large health plan. J Behav Health Serv Res. 2004;31(2): $117-133$.

4. Beck CT. A meta-analysis of the relationship between postpartum depression and infant temperament. Nurs Res. 1996;45(4):225-230.

5. Giallo R, Cooklin A, Nicholson JM. Risk factors associated with trajectories of mothers' depressive symptoms across the early parenting period: an Australian population-based longitudinal study. Arch Womens Ment Health. 2014;17:115-125.

6. Williamson V, McCutcheon H. Postnatal depression: a review of current literature. Aust J Midwifery. 2004;17(4):11-16.

7. Lee DTS, Chung THK. Postnatal depression: an update. Best Pract Res Clin Obstet Gynaecol. 2007;21(2):183-191.

8. Cooper P, Campbell EA, Day A, Kennerley H, Bond A. Non-psychotic psychiatric disorder after childbirth: a prospective study of prevalence, incidence, course and nature. Br J Psychiatry. 1988;152:799-806. 
9. American Psychiatric Association. Diagnostic and Statistical Manual of Mental Disorders. 5th ed. Washtington, DC: American Psychiatric Association; 2013.

10. Barnett B, Fowler C. Caring for the Family's Future: A Practical Workbook on Recognising and Managing Post-Natal Depression. Haymarket, NSW: Norman Swan Medical Communications; 1995.

11. Petrou S, Cooper P, Murray L, Davidson LL. Economic costs of postnatal depression in a high-risk British cohort. Br JPsychiatry. 2002;181: 505-512.

12. Leahy-Warren P, McCarthy G. Postnatal depression: prevalence, mothers' perspectives, and treatments. Arch Psychiatr Nurs. 2007;21(2): 91-100.

13. Reck C, Noe D, Gerstenlauer J, Stehle E. Effects of postpartum anxiety disorders and depression on maternal self-confidence. Infant Behav Dev. 2012;35(2):264-272.

14. Moehler E, Brunner R, Wiebel A, Reck C, Resch F. Maternal depressive symptoms in the postnatal period are associated with long-term impairment of mother-child bonding. Arch Womens Ment Health. 2006; 9(5):273-278.

15. Reck C, Noe D, Stefenelli U, et al. Interactive coordination of currently depressed inpatient mothers and their infants during the postpartum period. Infant Ment Health J. 2011;32(5):542-562.

16. Feldman R, Granat A, Pariente C, Kanety H, Kuint J, Gilboa-Schechtman E. Maternal depression and anxiety across the postpartum year and infant social engagement, fear regulation, and stress reactivity. J Am Acad Child Adolesc Psychiatry. 2009;48(9):919-927.

17. Hay DF, Pawlby S, Angold A, Harold GT, Sharp D. Pathways to violence in the children of mothers who were depressed postpartum. Dev Psychol. 2003;39(6):1083-1094.

18. Murray L, Halligan SL, Goodyer I, Herbert J. Disturbances in early parenting of depressed mothers and cortisol secretion in offspring: a preliminary study. J Affect Disord. 2010;122(3):218-223.

19. Letourneau NL, Dennis CL, Benzies K, et al. Postpartum depression is a family affair: addressing the impact on mothers, fathers, and children. Issues Ment Health Nurs. 2012;33(7):445-457.

20. Belsky J, Conger R, Cpaldi DM. The intergenerational transmission of parenting. Dev Psychol. 2009;45:1201-1204.

21. Manning C, Gregoire A. Effects of parental mental illness on children. Psychiatr J. 2006;5(1):10-12.

22. Meaney MJ. Maternal care, gene expression, and the transmission of individual differences in stress reactivity across generations. Annu Rev Neurosci. 2001;24:1161-1192.

23. Dennis CL, McQueen K. Does maternal postpartum depressive symptomatology influence infant feeding outcomes? Acta Paediatr. 2007; 96(4):590-594.

24. McLearn KT, Minkovitz CS, Strobino DM, Marks E, Hou W. Maternal depressive symptoms at 2 to 4 months postpartum and early parenting practices. Arch Pediatr Adolesc Med. 2006;160:279-284.

25. McLearn KT, Minkovitz CS, Strobino DM, Marks E, Hou W. The timing of maternal depressive symptoms and mothers' parenting practices with your children: implications for pediatric practice. Pediatrics. 2006;118:e174-e182.

26. Dennis CL, Ross L. Relationships among infant sleep patterns, maternal fatigue, and development depressive symptomatology. Birth. 2005; 32:187-193.

27. Hatton DC, Harrison-Hohner J, Dorato V, Curen LB, McCarron DA. Symptoms of postpartum depression and breastfeeding.J Hum Lact. 2005; 21:444-449.

28. Hiscock H, Wake M. Infant sleep problems and postnatal depression: a community-based study. Pediatrics. 2001;107:1317-1322.

29. Minkovitz CS, Strobino D, Charfstein D, Hou W, Miller T, Mistry KB. Maternal depressive symptoms and children's receipt of healthcare in the first 3 years of life. Pediatrics. 2005;115:306-314.

30. Zajicek-Farber ML. Postnatal depression and infant health practices among high-risk women. J Child Fam Stud. 2009;18(2):236-245.

31. Zajicek-Farber ML. The contributions of parenting and postnatal depression on emergent language of children in low-income families. J Child Fam Stud. 2010;19(3):257-269.
32. Tronick E, Reck C. Infants of depressed mothers. Harv Rev Psychiatry. 2009; 17(2):147-156.

33. Tronick E, Weinberg MK. Depressed mothers and infants: failure to form dyadic states of consciousness. In: Murray L, Cooper P, editors. Postpartum Depression and Child Development. New York, NY: Guilford Press; 1997:54-81.

34. Forman DR, O'Hara MW, Stuart S, Gorman LL, Larsen KE, Coy KC. Effective treatment for postpartum depression is not sufficient to improve the developing mother-child relationship. Dev Psychopathol. 2007;19(2):585-602.

35. McLennan JD, Offord DR. Should postpartum depression be targeted to improve child mental health? J Am Acad Child Adolesc Psychiatry. 2002;41(1):28-35.

36. O'Hara MW. Postpartum depression: what we know. J Clin Psychol. 2009;65(12):1258-1269.

37. Goodman SH, Gotlib IH. Risk for psychopathology in the children of depressed mothers: a developmental model for understanding mechanisms of transmission. Psychol Rev. 1999;106(3):458-490.

38. Murray L, Arteche A, Fearon P, Halligan S, Croudace T, Cooper P. The effects of maternal postnatal depression and child sex on academic performance at age 16 years: a developmental approach. J Child Psychol Psychiatry. 2010;51(10):1150-1159. [Research Support, Non-U.S. Gov't].

39. Murray L, Cooper P. Effects of postnatal depression on infant development. Arch Dis Child. 1997;77(2):99-101.

40. Murray L, Cooper P. The role of infant and maternal factors in postpartum depression, mother-infant interactions, and infant outcome. In: Murray LCPJ, editor. Postpartum Depression and Child Development. New York, NY: Guilford Press; 1997:111-135.

41. Grace SL, Evindar A, Stewart DE. The effect of postpartum depression on child cognitive development and behavior: a review and critical analysis of the literature. Arch Womens Ment Health. 2003;6(4):263-274.

42. Cohn JF, Campbell SB, Matias R, Hopkins J. Face-to-face interactions of postpartum depressed and nondepressed mother-infant pairs at 2 months. Dev Psychol. 1990;26(1):15-23.

43. Murray L, Arteche A, Fearon P, Halligan S, Goodyer I, Cooper P. Maternal postnatal depression and the development of depression in offspring up to 16 years of age. J Am Acad Child Adolesc Psychiatry. 2011;50(5):460-470.

44. Milgrom J, Westley D, Gemmill A. The mediating role of maternal responsiveness in some longer term effects of postnatal depression on infant development. Infant Behav Dev. 2004;27(4):443-454.

45. Kingston D, Tough S, Whitfield $H$. Prenatal and postpartum maternal psychological distress and infant development: a systematic review. Child Psychiatry Hum Dev. 2012;43:683-714.

46. Kingston D, Tough S. Prenatal and postnatal maternal mental health and school-age child development: a systematic review. Matern Child Health J. 2014;18:1728-1741.

47. Thompson RA. Early sociopersonality development. In: Damon W, Eisenberg N, editors. Handbook of Clinical Psychology. Vol. 3. New York: Wiley; 1998:25-104.

48. Stein A, Craske MG, Lehtonen A, et al. Maternal cognitions and mother-infant interaction in postnatal depression and generalized anxiety disorder. J Abnorm Psychol. 2012;121(4):795-809.

49. Crittenden PM. Raising Parents: Attachment, Parenting and Child Safety. Devon: Willan; 2008.

50. Gerhardt S. Why love matters: How Affection Shapes a Baby's Brain. New York: Routledge/Taylor and Francis Group; 2004.

51. Hay DF. Postpartum depression and cognitive development. In: Murray L, Cooper PJ, editors. Postpartum Depression and Child Development. New York: Guilford Press; 1997:85-110.

52. Moutsiana C, Fearon P, Murray L, et al. Making an effort to feel positive: insecure attachment in infancy predicts the neural underpinnings of emotion regulation in adulthood. J Child Psychol Psychiatry. 2014;55(9):999-1008.

53. Arteche A, Joorman J, Harvey A, et al. The effects of postnatal maternal depression and anxiety on the processing of infant faces. $J$ Affect Disord. 2011;133:197-203. 
54. Van Den Boom DC. The influence of temperament and mothering on attachment and exploration: an experimental manipulation of sensitive responsiveness among lower-class mothers with irritable infants. Child Dev. 1994;65(5):1457-1477.

55. Logsdon M, Wisner K, Hanusa BH. Does maternal role functioning improve with antidepressant treatment in women with postpartum depression? J Womens Health. 2009;18(1):85-90.

56. Pearlstein TB, Zlotnick C, Battle CL, et al. Patient choice of treatment for postpartum depression: a pilot study. Arch Womens Ment Health. 2006;9(6):303-308.

57. Austin MP, Frilingos M, Lumley J, et al. Brief antenatal cognitive behaviour therapy group intervention for the prevention of postnatal depression and anxiety: a randomised controlled trial. J Affect Disord. 2008;105(1-3):35-44.

58. Rojas G, Fritsch R, Solis J, et al. Treatment of postnatal depression in low-income mothers in primary-care clinics in Santiago, Chile: a randomised controlled trial. Lancet. 2007;370(9599): $1629-1637$.

59. Ugarriza DN. Group therapy and its barriers for women suffering from postpartum depression. Arch Psychiatr Nurs. 2004;18(2): 39-48.

60. Milgrom J, Ericksen J, McCarthy R, Gemmill AW. Stressful impact of depression on early mother-infant relations. Stress Health. 2006;22(4): 229-238.

61. Murray L, Cooper PJ, Wilson A, Romaniuk H. Controlled trial of the short- and long-term effect of psychological treatment of post-partum depression 2. Impact on the mother-child relationship and child outcome. Br J Psychiatry. 2003;182:420-427.

62. Mulcahy R, Reay RE, Wilkinson RB, Owen C. A randomised control trial for the effectiveness of group interpersonal psychotherapy for postnatal depression. Arch Womens Ment Health. 2010;13(2):125-139.

63. Reay R, Fisher Y, Robertson M, Adams E, Owen C. Group interpersonal psychotherapy for postnatal depression: a pilot study. Arch Womens Ment Health. 2006;9(1):31-39.

64. Van Doesum K, Riksen-Walraven J, Hosman C, Hoefnagels C. A randomized controlled trial of a home-visiting intervention aimed at preventing relationship problems in depressed mothers and their infants. Child Dev. 2008;79(3):547-561.

65. Clark R, Tluczek A, Brown R. A mother-infant therapy group model for postpartum depression. Infant Ment Health J. 2008;29(5):514-536.

66. Jung V, Short R, Letourneau N, Andrews D. Interventions with depressed mothers and their infants: modifying interactive behaviours. J Affect Disord. 2007;98(3):199-205.

67. O’Higgins M, Roberts IS, Glover V. Postnatal depression and mother and infant outcomes after infant massage. J Affect Disord. 2008; 109(1-2):189-192.

68. Onozawa K, Glover V, Adams D, Modi N, Kumar RC. Infant massage improves mother-infant interaction for mothers with postnatal depression. J Affect Disord. 2001;63(1-3):201-207.

69. Nylen KJ, Moran TE, Franklin CL, O’Hara MW. Maternal depression: a review of relevant treatment approaches for mothers and infants. Infant Ment Health J. 2006;27(4):327-343.

70. Cuijpers P, Brannmark JG, Van Straten A. Psychological treatment of postpartum depression: a meta-analysis. J Clin Psychol. 2008;64(1): 103-118.

71. Tandon SD, Perry DF, Mendelson T, Kemp K, Leis JA. Preventing perinatal depression in low-income home visiting clients: a randomized controlled trial. J Consult Clin Psychol. 2011;79(5):707-712.

72. Morrell CJ. Review of interventions to prevent or treat postnatal depression. Clin Eff Nurs. 2006;9(2):e135-e161.

73. Claridge AM. Efficacy of systematically oriemted psychotherapies in the treatment of perinatal depression: a meta-analysis. Arch Womens Ment Health. 2014;17:3-15.

74. Poobalan AS, Aucott LS, Ross L, Smith WCS, Helms PJ, Williams JHG. Effects of treating postnatal depression on mother-infant interaction and child development: systematic review. Br J Psychiatry. 2007;191(4): $378-386$.
75. Tarrier N, Wykes $\mathrm{T}$. Is there evidence that cognitive behaviour therapy is an effective treatment for schizophrenia? A cautious or cautionary tale? Behav Res Ther. 2004;42(12):1377-1401.

76. Jadad AR, Moore RA, Carroll D, et al. Assessing the quality of reports of randomized clinical trials: is blinding necessary? Control Clin Trials. 1996;17(1):1-12

77. Cohen J. Statistical Power Analysis for the Behavioral Sciences. 2 ed. Hillsdale: Lawrence Erlbaum Associates, Inc; 1988.

78. Clark R, Tluczek A, Wenzel A. Psychotherapy for postpartum depression: a preliminary report. Am J Orthopsychiatry. 2003;73(4):441-454.

79. Field T, Grizzle N, Scafidi F, et al. Massage therapy for infants of depressed mothers. Infant Behav Dev. 1996;19(1):107-112.

80. Horowitz JA, Bell M, Trybulski J, et al. Promoting responsiveness between mothers with depressive symptoms and their infants. J Nurs Scholarsh. 2001;33(4):323-329.

81. Kersten-Alvarez LE, Hosman CMH, Riksen-Walraven JM, Van Doesum KTM, Hoefnagels C. Long-term effects of a home-visiting intervention for depressed mothers and their infants. J Child Psychol Psychiatry. 2010;51(10):1160-1170.

82. Cooper P, Murray L, Wilson A, Romaniuk H. Controlled trial of the short- and long-term effect of psychological treatment of post-partum depression. 1. Impact on maternal mood. Br J Psychiatry. 2003; 182(5):412-419.

83. Beeber LS, Schwartz TA, Holditch-Davis D, Canuso R, Lewis V, Wilde Hall H. Parenting enhancement interpersonal psychotherapy to reduce depression in low-income mothers of infants and toddlers: a randomised trial. Nurs Res. 2013;62:82-90.

84. Horowitz JA, Murphy CA, Gregory K, Wojcik J, Pulcini J, Solon L. Nurse home visits improve maternal/infant interaction and decrease severity of postpartum depression. J Obstet Gynecol Neonatal Nurs. 2013;42:287-300

85. Goodman JH, Prager J, Goldstein R, Freeman M. Perinatal dyadic psychotherapy for postpartum depression: a randomised controlled pilot trial. Arch Womens Ment Health. In press 2014.

86. Tsivos ZL, Calam R, Sanders MR, Wittkowski A. A pilot randomised controlled trial to evaluate the feasibility and acceptability of the baby triple $P$ positive parenting programme in mothers with postnatal depression. Clin Child Psychol Psychiatry. In press 2014:1-23.

87. Clark R. The Parent-Child Early Relational Assessment. [Unpublished instrument]. Department of Psychiatry, University of Wisconsin; 1985.

88. Richman N, Graham P. A behavioural screening questionnaire for use with three-year old children, preliminary findings. J Child Psychol Psychiatry. 1971;12(1):5-33.

89. Goodman SH, Broth MR, Hall CM, Stowe ZN. Treatment of postpartum depression in mothers: secondary benefits to the infants. Infant Ment Health J. 2008;29(5):492-513.

90. Boath EH, Henshaw C. The treatment of postnatal depression: a comprehensive literature review. J Reprod Infant Psychol. 2001;19(3):215-248.

91. Dennis CL. Can we identify mothers at risk for postpartum depression in the immediate postpartum period using the Edinburgh postnatal depression scale? J Affect Disord. 2004;78(2):163-169.

92. Righetti-Veltema M, Conne-Perreard E, Bousquet A, Manzano J. Postpartum depression and mother-infant relationship at 3 months old. J Affect Disord. 2002;70(3):291-306.

93. Gameiro S, Moura-Ramos M, Canavarro MC. Maternal adjustment to the birth of a child: primiparity versus multiparity. J Reprod Infant Psychol. 2009;27(3):269-286.

94. Carter AS, Briggs-Gowan MJ, Jones SM, Little TD. The infant-toddler social and emotional assessment (ITSEA): factor structure, reliability, and validity. J Abnorm Child Psychol. 2003;31(5):495-514.

95. Goodman JH. Paternal postpartum depression, its relationship to maternal postpartum depression, and implications for family health. $J A d v$ Nurs. 2004;45(1):26-35.

96. Paulson J, Bazemore S. Prenatal and postpartum depression in fathers and its association with maternal depression: a meta-analysis. JAMA. 2010;303(19):1961-1969. 
97. Kowlessar O, Fox JRE, Wittkowski A. The pregnant male: a metasynthesis of fathers' experiences of pregnancy. J Reprod Infant Psychol. 2015;33(2):106-127.
98. Sharma V, Mazmanian D. The DSM-5 peripartum specifier: prospects and pitfalls. Arch Womens Ment Health. 2014;17:171-173.

\section{Publish your work in this journal}

The International Journal of Women's Health is an international, peerreviewed open-access journal publishing original research, reports, editorials, reviews and commentaries on all aspects of women's healthcare including gynecology, obstetrics, and breast cancer. The manuscript management system is completely online and includes a very quick and fair peer-review system, which is all easy to use. Visit http://www.dovepress.com/testimonials.php to read real quotes from published authors. 